\title{
Comparação dos índices de eficiência reprodutiva por diferentes métodos em rebanhos bovinos leiteiros
}

\author{
[Comparison of reproductive efficiency indexes by different methods \\ in dairy cattle herds] \\ P.A.C. Pereira ${ }^{1}$, A.M. Ferreira ${ }^{2}$, L.B. Carvalho ${ }^{*}$, R.S. Verneque ${ }^{2}$, \\ M. Henry ${ }^{3}$, R.C. Leite ${ }^{3}$ \\ ${ }^{1}$ Aluno de pós-graduação - Escola de Veterinária - Universidade Federal de Minas Gerais - Belo Horizonte, MG \\ ${ }^{2}$ Embrapa Gado de Leite - Juiz de Fora, MG \\ ${ }^{3}$ Escola de Veterinária - Universidade Federal de Minas Gerais - Belo Horizonte, MG
}

\section{RESUMO}

O presente estudo teve como objetivo promover o levantamento de índices zootécnicos para calcular e comparar a eficiência reprodutiva de rebanhos bovinos leiteiros por diferentes métodos. O intervalo de partos dos rebanhos estudados apresentou uma variação de 13,3 a 15,2 meses por meio dos diferentes métodos utilizados para sua obtenção, não se observando diferença significativa $(\mathrm{P}>0,05)$ entre eles. As avaliações pelos métodos de Johnson et al. (1964) e Butendieck et al. (1972) mostraram, respectivamente, eficiência reprodutiva de 79,5 e 77,8\%. Embora considerados métodos mais eficientes que o de Willcox et al. (1957), cuja média encontrada para os mesmos rebanhos foi de $90,2 \%$, não se observou diferença significativa entre os resultados obtidos pelos três métodos.

Palavras-chave: bovinos, índices, eficiência reprodutiva

\begin{abstract}
The study aimed to promote the lifting of indexes to calculate and compare the reproductive efficiency of dairy cattle herds by different methods. The parturition interval studied showed a variation from 13.3 to 15.2 months by means of different methods for obtaining it are not observing a significant difference $(P>$ 0.05) between them. Evaluations by the methods of Johnson et al (1964) and Butendieck et al. (1972) showed, respectively, reproductive efficiency of 79.5 and $77.8 \%$. Although regarded as the most efficient methods of Willcox et al. (1957), whose average found in the same herd was 90.2\%, there was no significant difference between the results obtained by three methods.
\end{abstract}

Keywords: bovine, indexes, reproductive efficiency

\section{INTRODUÇÃO}

O processo produtivo da pecuária bovina fundamenta-se, primariamente, na eficiência reprodutiva dos rebanhos (MacMillan, 1992; Hansen e Aréchiga, 2003). Uma adequada eficiência reprodutiva dos bovinos é essencial para maximizar a produção de leite e de carne, com o parto sendo o evento reprodutivo de maior significado em um sistema de produção, pela geração de uma nova cria e início de uma lactação (Neves et al., 1999).

Recebido em 22 de novembro de 2011

Aceito em 13 de março de 2013

*Autor para correspondência (corresponding author)

E-mail: carvalho_vet@yahoo.com.br
A performance reprodutiva é um ponto crítico para a lucratividade de um sistema de produção leiteiro, por determinar a taxa de eliminação e o número de animais para reposição, o progresso genético, a duração do período seco e a produção leiteira do rebanho (Stevenson, 1996b; Gröhn et al., 2000; Ferreira, 2002; Pereira et al., 2004). Contudo, para a obtenção de padrões ideais de eficiência reprodutiva, é preciso que ocorra perfeita interação dos parâmetros genéticos, reprodutivos, sanitários e nutricionais, fazendose necessária a análise da eficiência animal, com o estabelecimento e a avaliação de parâmetros e 
índices reprodutivos, para que se possa identificar e definir metas, bem como monitorar e solucionar os fatores que estão comprometendo a reprodução e a produção do rebanho (Sowden, 1990; Ferreira, 1994; Stevenson, 1996a; Sartori, 2007).

A eficiência reprodutiva do rebanho bovino pode ser mensurada por meio da obtenção de diversos índices, sendo o intervalo de partos (IP), as taxas de prenhez e de natalidade, além do período de serviço os mais utilizados (Varner e Majeskie, 1988; Faria, 1991; Stevenson, 1994; Ferreira et al., 1997; Pereira, 2005). Embora não muito utilizados, outros métodos mais complexos são descritos como formas mais precisas de se medir a eficiência reprodutiva de rebanhos leiteiros. Alguns desses métodos apresentam vantagens em relação aos índices mais consagrados por incluírem na avaliação animais com problemas reprodutivos e matrizes com um só parto durante o período de tempo avaliado (Wilcox et al.,1957; Johnson et al., 1964; Butendieck et al., 1972).

Pode-se obter o IP com base nas anotações zootécnicas existentes na propriedade, principalmente as datas de parição das matrizes. Contudo, estima-se que no Brasil uma pequena parcela de produtores realiza essa prática, o que dificulta os processos de avaliação. Na ausência de anotações, é possível realizar uma estimativa do IP baseando-se em outros índices, como as taxas de prenhez e de natalidade. Sabe-se que esses índices nos valores de, respectivamente, $75 \%$ e $100 \%$ correspondem a 12 meses de IP (Ferreira et al., 1997). Em rebanhos leiteiros, pode-se maximizar a produção de leite e de bezerros com IP em torno de 12 a 13 meses, período considerado ideal para se obter melhor retorno econômico e zootécnico na atividade, desde que utilizado um manejo racional (Ferreira, 2002; Pereira et al., 2004b).

Levantamentos realizados nos estados de Minas Gerais (Carneiro, 1992) e Rio de Janeiro (Ferreira et al., 1997) possibilitaram calcular a média do IP dos rebanhos estudados de 18 a 19 meses. Pereira et al. (2004), ao estudarem propriedades de exploração leiteira na região do Vale do Paraíba-SP, encontraram 16,5 meses de IP médio em rebanhos de fornecedores da
Cooperativa de Laticínios de Guaratinguetá. Tais resultados indicaram que a eficiência reprodutiva dos rebanhos dessas regiões, consideradas as mais desenvolvidas do país, está abaixo do ideal com base no longo IP encontrado, o que possibilita concluir que, nas regiões menos favorecidas do país, o IP seja igual ou até mesmo superior, impedindo a exploração do real potencial produtivo das matrizes leiteiras.

O método de Wilcox et al. (1957) é uma medida complexa de avaliação da eficiência reprodutiva, que tem como base o intervalo de partos e foi elaborado para medir a herdabilidade da eficiência reprodutiva, possuindo, portanto, as mesmas limitações citadas para o IP.

O método de avaliação de Johnson et al. (1964) possibilita avaliar a eficiência reprodutiva de um rebanho a qualquer momento, condição que o coloca em vantagem quando comparado àqueles que só contemplam as vacas que parem dentro de um determinado período. Esse método é mais preciso na estimativa da eficiência reprodutiva, mas é mais baseado nas falhas reprodutivas do que nos êxitos. Possui a desvantagem de ser restrito em considerar as novilhas recémintroduzidas no rebanho e que não pariram no momento do estudo, bem como de permitir medir a eficiência reprodutiva do rebanho apenas em um certo momento ou período curto, mas não em intervalos ou períodos mais longos.

O método contínuo de Butendieck et al. (1972) considera como normal uma vaca que tenha um bezerro por ano, ou seja, um IP de 365 dias significando $100 \%$ de fertilidade, empregando-se o conceito dias/vaca gestante (DVG) e dias/vaca excesso (DVE). Os DVG se referem aos dias de gestação que terminam com um bezerro nascido. OS DVE contabilizam somente aqueles animais que ingressaram posteriormente ou que foram eliminados durante o período de avaliação. Desta forma, considera-se o tempo de permanência real dos animais no período de estudo.

O presente estudo teve como objetivos realizar um levantamento de índices zootécnicos e comparar a eficiência reprodutiva dos rebanhos estudados por diferentes métodos convencionais e complexos. 


\section{MATERIAL E MÉTODOS}

O trabalho foi conduzido utilizando-se informações de rebanhos localizados na bacia leiteira de Guaratinguetá, região do Vale do Paraíba, estado de São Paulo, sudeste do Brasil. Oito rebanhos leiteiros, com animais de composição genética variando entre 1/2 Holândês/Gir e 15/16 Holandês/Gir, foram selecionados e, posteriormente, monitorados mensalmente de janeiro de 2006 a dezembro de 2007. Os rebanhos escolhidos foram agrupados em função do tamanho, de modo que o grupo 1 foi composto por rebanhos com até 50 vacas; o grupo 2 composto por rebanhos que continham entre 50 e 100 vacas; e o grupo 3 constituído por rebanhos acima de 100 vacas. A idade dos animais estudados variou de $18 \mathrm{a} \geq 60$ meses.

Durante o estudo, foram efetuados levantamentos de índices zootécnicos, como as taxas de prenhez, de natalidade, o período de serviço e o intervalo de partos; bem como foram acompanhados e notificados todos os casos de problemas reprodutivos (abortamentos, retenção de placenta, infecções uterinas, cistos ovarianos e anestro) ocorridos no transcorrer do estudo. Essas informações foram importantes para a obtenção do número de dias gestantes ou não gestantes de cada animal, indispensável para o cálculo de alguns dos índices estudados.
Foi calculada a taxa média anual para cada um dos índices, que serviu de base para os cálculos do IP a partir de cada um deles. Os resultados foram comparados entre si e com o IP obtido pelas anotações das datas de parto de cada vaca. Além disso, foi calculado o período de serviço das matrizes, o que serviu como base para o cálculo do intervalo de partos projetado. Também foi medida a eficiência reprodutiva dos rebanhos por meio da utilização dos métodos complexos de Wilcox et al. (1957), Johnson et al. (1964) e Butendieck et al. (1972), comparando-se os resultados obtidos.

\section{RESULTADOS E DISCUSSÃO}

Os resultados dos índices reprodutivos dos rebanhos bovinos estudados são apresentados na Tab. 1, onde se verifica, com base nas anotações das datas do parto, que dos oito rebanhos apenas um $(9,0 \%)$ apresentou IP entre 12 e 13 meses, período considerado ideal para maximizar a rentabilidade da atividade leiteira em rebanhos bovinos (Sowden, 1990; Ferreira, 1994; Stevenson, 1996a ). Observa-se também que os demais sete rebanhos $(91,0 \%)$ apresentaram IP variando entre 13,1 e 14,1 meses, sendo classificados como rebanhos com problema leve a moderado (Varner e Majeskie, 1988).

Tabela 1. Taxa de natalidade (\%), taxa de vacas em lactação (\%), taxa de prenhez (\%), intervalo de partos (meses), intervalo de partos projetado (meses) e período de serviço (dias), em três grupos (g) de oito rebanhos bovinos leiteiros.

\begin{tabular}{cccccccccccc}
\hline Gr & Reb & Vacas & TP & IP1 & TN & IP2 & TVL & IP3 & IP4 & PS & IPP \\
\hline \multirow{3}{*}{1} & A & 18 & 61,1 & 14,7 & 84,8 & 14,2 & 69,3 & 14,4 & 13,6 & 149 & 14,1 \\
& B & 13 & 55,5 & 16,2 & 80,2 & 15,0 & 61,5 & 16,2 & 14,1 & 169 & 14,8 \\
& C & 34 & 66,2 & 13,6 & 71,2 & 16,9 & 58,7 & 17,0 & 13,1 & 225 & 16,6 \\
\hline \multirow{2}{*}{2} & D & 75 & 63,8 & 14,1 & 86,2 & 13,9 & 71,5 & 13,9 & 13,3 & 135 & 13,6 \\
& $\mathrm{E}$ & 65 & 67,2 & 13,3 & 85,2 & 14,1 & 72,0 & 13,8 & 13,6 & 139 & 13,8 \\
& $\mathrm{~F}$ & 57 & 46,8 & 19,2 & 69,8 & 17,2 & 54,8 & 18,2 & 13,7 & 237 & 17,0 \\
\hline \multirow{2}{*}{3} & $\mathrm{G}$ & 153 & 82,5 & 11,0 & 98,1 & 12,2 & 78,3 & 12,7 & 11,9 & 91 & 12,2 \\
& $\mathrm{H}$ & 117 & 49,5 & 18,8 & 77,6 & 15,5 & 64,5 & 15,4 & 13,4 & 181 & 15,2 \\
\hline \multicolumn{2}{c}{ Média } & 66,5 & 61,6 & $15,1 \mathrm{a}$ & 81,6 & $14,9 \mathrm{a}$ & 66,3 & $15,2 \mathrm{a}$ & $13,3 \mathrm{a}$ & 165,7 & $14,7 \mathrm{a}$ \\
\hline
\end{tabular}

* Médias seguidas por letras iguais na linha não diferem estatisticamente $(\mathrm{P}>0,05)$.

$\mathrm{TN}=$ taxa de natalidade/TVL $=$ taxa de vacas em lactação/TP= taxa de prenhez obtida de matrizes com um ou mais partos/IP1 = intervalo de partos obtido por meio da TP/IP2 = intervalo de partos obtido por meio da TN/IP3 = intervalo obtido por meio da TVL/ IP4 = intervalo de partos obtido por meio das anotações das datas dos partos/IPP = intervalo de partos projetado/PS= período de serviço.

$\mathrm{Gr}=$ grupo (1: até 50 vacas; $2: 50$ a 100 vacas; e 3: mais de 100 vacas). Reb $=$ Rebanho. 
O intervalo de partos médio (IPM) dos rebanhos estudados foi de 13,3 meses, acima dos 12 meses preconizados para rebanhos manejados com eficiência, mas, ainda assim, bastante inferior aos de 18 a 19 meses citados como a média da maioria dos rebanhos leiteiros do Brasil (Carneiro, 1992; Ferreira et al., 1997; Pereira et al., 2004). Isso é explicado pelo fato de as propriedades estudadas estarem localizadas na região do Vale do Paraíba-SP, local de pecuária leiteira tradicional, além de todas possuírem assistência técnica mensal, realidade que as possibilita serem conduzidas de maneira mais eficiente do que as demais da própria região e de outras localidades do país, inclusive com as anotações zootécnicas que possibilitam o presente estudo, o que não é comum na pecuária leiteira nacional.

Em razão das limitações do IP como medida de eficiência de rebanho, por não incluir matrizes com um só parto e aquelas com problemas reprodutivos durante o período avaliado, foi calculado o período de serviço (PS) dos rebanhos estudados. Sabe-se que, para obter IP entre 12 e 13 meses, é necessário que o PS de um rebanho varie de 85 a 120 dias. Na Tab. 1 fica evidente que, à exceção do rebanho $\mathrm{G}$, que apresentou PS de 91 dias, os demais rebanhos apresentaram esse índice acima dos 120 dias.

Neste estudo, o PS apresentou média de 165,7 dias entre os oito rebanhos, o que, segundo Esslemont (1993), representa um problema grave. Esse mesmo autor considera ideal um PS que varie entre 75 e 85 dias, para que o IP alcançado seja de 12 meses. O aumento desse índice provavelmente foi reflexo da participação dos distúrbios puerperais. Pode-se verificar em quase todos os rebanhos que, apesar de as infecções uterinas e de a retenção de placenta terem sido diagnosticadas, o anestro ou aumento do tempo de retomada da atividade ovariana luteal cíclica (AOLC) pós-parto mostrou-se a causa mais frequente. Segundo Ferreira et al. (1997), a condição corporal inferior ao parto e o déficit nutricional nas semanas subsequentes são as principais causas do longo IP verificado em rebanhos distribuídos por todo o país. Outras causas, como estresse e amamentação, podem interferir na fertilidade e no retorno da AOLC pós-parto, uma vez que a produção de opioides endógenos nessas situações resulta em menores taxas de crescimento folicular (Ferreira, 1990). Em seis dos oito rebanhos estudados, em razão de as matrizes serem mestiças, a ordenha era feita com a presença de bezerros para o estímulo da "descida do leite", o que pode ter contribuído para o maior PS verificado.

Com base no PS, foi possível realizar o cálculo do intervalo de partos projetado (IPP), eliminando-se as distorções no resultado da eficiência reprodutiva de um rebanho em função das limitações do IP, embora não fossem considerados possíveis abortamentos futuros. Dessa maneira, pode-se verificar uma variação no IPP dos rebanhos estudados de 12,2 a 17,0 meses. Mesmo se observando um aumento da média do IPP em relação ao IP obtido por meio das anotações de datas dos partos, essa elevação não foi considerada significativa $(P>0,05)$.

Verifica-se, na Tab. 1, que a média do IP dos rebanhos obtida pelos diversos índices apresentou uma variação de 13,3 a 15,2 meses. Mesmo sendo observado um resultado inferior do IP obtido por meio das anotações de datas do parto com as demais formas utilizadas, não foi observada uma diferença significativa $(P>0,05)$ entre os resultados obtidos neste estudo. Entretanto, sabe-se que existe uma correlação entre elevação do IP e redução na produção leiteira da ordem de $8,33 \%$ para cada mês aumentado de 12 meses (Ferreira, 2002). Neste caso, quando da avaliação das perdas decorrentes da menor eficiência, seria observada uma redução de $10,8 \%$ na produção leiteira quando utilizado o IP de 13,3 meses e de $26,6 \%$ com IP de 15,2 meses, representando uma diferença de $15,8 \%$. Desta maneira, pode-se sugerir que a utilização do intervalo de partos projetado (IPP), por se tratar do valor mais próximo da real eficiência reprodutiva, quando da utilização desse índice para a avaliação, mostra-se mais adequado para a avaliação do rebanho e das eventuais perdas decorrentes da sua menor eficiência. 
Tabela 2. Eficiência reprodutiva obtida pelos métodos complexos de Wilcox et al. (1957), Johnson et al. (1964) e Butendieck et al. (1972), intervalo de partos (IP) e intervalo de partos projetado (IPP) de oito rebanhos bovinos leiteiros

\begin{tabular}{ccccccc}
\hline \multirow{2}{*}{ Grupo } & Rebanho & Vacas & WLC (\%) & JHN (\%) & BTD (\%) & IP (meses) \\
\hline \multirow{2}{*}{1} & A & 18 & 88,4 & 85,7 & 78,5 & 13,6 \\
& B & 13 & 85,1 & 81,5 & 76,1 & 14,1 \\
& C & 34 & 91,9 & 86,6 & 83,2 & 13,1 \\
\hline \multirow{2}{*}{2} & D & 75 & 90,4 & 78,4 & 72,6 & 13,3 \\
& E & 65 & 88,0 & 77,9 & 77,5 & 13,6 \\
\hline 3 & F & 57 & 87,7 & 69,5 & 59,7 & 13,7 \\
\hline & G & 153 & 101,2 & 90,2 & 97,4 & 11,9 \\
\hline
\end{tabular}

* Médias seguidas por letras iguais na linha não diferem estatisticamente $(\mathrm{P}>0,05)$.

WLC = eficiência reprodutiva pelo método de Willcox et al. $(1957) / J H N=$ eficiência reprodutiva pelo método de Johnson et al. (1964)/BTD = eficiência reprodutiva pelo método de Buttendieck et al. (1972)/IP = intervalo de partos obtido por meio das anotações das datas dos partos/IPP = intervalo de partos projetado.

A média encontrada entre a eficiência reprodutiva dos rebanhos avaliada pelo método de Willcox et al. (1957) foi de 90,2 \%,variando de 85,1 a $101,2 \%$. A utilização desse método isoladamente não se mostrou a maneira mais adequada para a avaliação dos rebanhos, uma vez que é uma medida correlacionada com o IP, apresentando, portando, as mesmas limitações desse índice por não incluir na avaliação vacas com um só parto durante o período avaliado e aquelas com problemas reprodutivos. Desta maneira, fica evidente que a eficiência reprodutiva calculada por esse método aumenta à medida que o IP diminui.

A avaliação da eficiência reprodutiva pelos métodos de Johnson et al. (1964) e Butendieck et al. (1972) mostrou, respectivamente, 79,5 e $77,8 \%$ como resultados médios entre os rebanhos estudados. Embora sejam considerados métodos mais eficientes que o Willcox et al. (1957), não foram observadas diferenças significativas entre os resultados obtidos pelos três métodos. Contudo, sugere-se que o método de Butendieck et al. (1972) seja mais eficiente que as demais maneiras utilizadas neste estudo para avaliar os rebanhos, por se tratar de um método que considera o tempo de permanência das matrizes no rebanho, assim como o tempo em que elas ficaram gestantes, considerando somente as gestações completas, durante o período avaliado.

\section{CONCLUSÕES}

A utilização de métodos mais complexos de medidas como índice de eficiência reprodutiva mostrou-se altamente satisfatória na avaliação dos rebanhos. O método de Butendieck et al. (1972) apresentou o menor número de limitações para estimação do índice do intervalo de partos de rebanhos leiteiros.

\section{AGRADECIMENTOS}

Os autores agradecem ao INCT-Pecuária: CNPq proc. 573899/2008-8 e FAPEMIG APQ-0084/08.

\section{REFERÊNCIAS}

BUTENDIECK, N.; STEHR, G.; BONILLA, M.V. Metodo contínuo para determinar la Eficiencia Reprodutiva y tasa de incremento annual en una poblacion bovina. Arch. Med. Vet., v.4, p.26-30, 1972.

CARNEIRO, J.M. Principais problemas da bovinocultura de leite : o caso de MG. Belo Horizonte : Fundação João Pinheiro, 1992. 219p.

ESSLEMONT, R.J. Relationship between herd calving to conception interval and culling rate for failure to conceive. Vet. Record, v.133, p.163164, 1993.

FARIA, V.P. Estrutura atual de produção de leite no Brasil. In: NESTLÉ. 4ํㅡ Curso de pecuária leiteira. ESALQ. Piracicaba, 1991. p.19-22. 
FERREIRA, A.M. Intervalo de partos reduzido, aumento da eficiência do rebanho. G. Holandês, v.429, p.40-45, 1994.

FERREIRA, A.M. Como reduzir o intervalo entre partos. DBO Especial Mundo do Leite. v.1, p.32-33, 2002.

FERREIRA, A.M.; TEIXEIRA, S.R.; SANTOS, P.C.B. et al. Taxa de natalidade em rebanhos leiteiros do Estado do Rio de Janeiro. Rev. Bras. Reprod. Anim., v.21, p.122-124, 1997.

GRÖHN, Y.T.; RAJALA-SCHULTZ, P.J. Epidemiology of reproductive performance in dairy cows. Anim. Reprod. Sci., v.60-61, p.605$614,2000$.

HANSEN, P.J.; ARÉCHIGA, C.F. Estratégias para reduzir os efeitos do estresse térmico na eficiência reprodutiva. IN: Novos Enfoques na Produção e Reprodução de Bovinos, 7., 2003. Uberlândia- MG, Anais.... Uberlandia:Unesp. 2003. p.77-97.

JOHNSSON, A.O.; MYERS, R.M.; ULBERG, L.C. A method for evaluating the current reproductive status of a dairy herd. J. Am. Vet. Med. Assoc., v.144, p.994-997, 1964.

MAcMILLAN, K.L. Reproductive Management. In: VAN HORN, H.H.; WILCOX, C.J. Large Dairy Herd Management. EUA: Champaign, 1992. p.88-98.

NEVES, J.P.; GONÇALVES, P.B.D.; OLIVEIRA, J.F.C. Fatores que afetam a eficiência reprodutiva na vaca. Rev. Bras. Reprod. Anim., v.23, p.105, 1999.

PEREIRA, P.A.C. Impacto de doenças reprodutivas e do manejo sobre a produção $e$ reprodução em rebanhos bovinos leiteiros na região de Guaratinguetá-SP. 2005. $50 \mathrm{f}$. Dissertação (Mestrado) - Escola de Veterinária. Universidade Federal de Minas Gerais. Belo Horizonte.
PEREIRA, P.A.C.; FERREIRA, A.M.; CARVALHO, L.B. et al. Estimativa de Perdas na Produção de Leite devido ao aumento do Intervalo de Partos de rebanhos bovinos do Vale do Paraíba-SP. Rev. Bras. Reprod. Anim., v.28, p.233-236, 2004.

SARTORI, R. Manejo Reprodutivo da fêmea leiteira. Reprod Anim., v.31, p.153-159, 2007.

STEVENSON, J. Measure and understand reproductive efficiency. Hoard's Dairyman, v.139, p.774, 1994.

STEVENSON, J. Is there an optimal calving interval ? Hoard's Dairyman, v.141, p.408, 1996a .

STEVENSON, J. Dissecting a calving interval. Hoard's Dairyman, v.141, p.326, 1996 b.

SOWDEN, C.L. Culling economics. Dairy Herd Managem., v.27, p.22-24, 1990.

VARNER, M.A.; MAJESKIE, J.L. Interpreting Indexes of Reproductive Efficiency. Natural Cooperative Dairy Herd Improvement Program. Handbook. p.1-6. 1988.

WILCOX, C.S.; PFAU, K.O.; BARTIET, J.W. An investigation of the inheritance and longevity and their interrelationships within a holsteinfriesian herd. J. Dairy Sci., v.40, p.942-946, 1957. 